\title{
Ein Beitrag zum Kapitel: Dermatologische Kriegserfahrungen.
}

\author{
Von Dr. J. F. v. Crippa-Linz, Bad Hall.
}

\section{$A$. Künstlich hervorgerufene Hautkrankheiten.}

Der Krieg hat wie auf allen Gebieten, so auch auf dem Gebiete der Medizin eine einseitige Verschiebung, oder, wie der technische Ausdruck lautet, eine Umwertung bewirkt. Chirurgie und Seuchenlehre sind heute Trumpf. Doch auch die andern Disziplinen unsrer Wissenschaft arbeiten, wenn auch weniger im Vordergrund und weniger beachtet, nach besten Kräften und erfolgreich an der Verwirklichung des hohen Zieles mit, unsre Wehrfähigkeit und damit die Aussicht auf den erhofften Sieg zu festigen. Verwundete und Kranke werden nach den modernsten und bewährtesten Grundsätzen rasch und gründlich wieder felddiensttauglich gemacht. Mit Genugtuung und Stolz können wir da feststellen, daß wir auch in diesem Punkte moderner Kriegführung unsre Gegner überragen. Eine hehre und vaterländische Aufgabe! Unser Beruf bringt es aber mit sich, daß wir nicht nur in diesem Sinne dem Staate und unsrem Volke Dienste leisten, wir müssen auch weniger erhebende Arbeit ausführen, das ist, ich möchte sagen, der Kampf mit den Drückebergern. Wir sind zwar auf diesem Gebiete besser daran wie unsre Gegner, z. B. die Engländer, doch gibt es naturgemäß auch bei uns Einzelindividuen, die sich der Kriegspflicht entziehen wollen. Diese Ehrenmänner rekrutieren sich aus allen Volksschichten; die versuchten Ausflüchte sind dementsprechend äußerst mannigfaltig, lassen sich aber zwanglos in zwei Gruppen teilen. Wir wollen uns nur mit der Gruppe befassen, welche körperliche Gebrechen vorschützt, um dem Schützengraben zu entgehen. Es soll hier nur von jenen Individuen gesprochen werden, die sich zur Befreiung vom Militär- oder doch wenigstens Frontdienst ein körperliches 
Leiden künstlich hervorrufen. Wir beschränken uns in den folgenden Ausführungen auf die Gruppe der künstlich hervorgerufenen Hautkrankheiten. Aus der vorliegenden Literatur ist diesbezüglich wenig zu ersehen. Die Lehrbücher berühren nur kurz dieses Kapitel; sie sind ja alle in friedlichen Verhältnissen und für das Studium zn Friedenszeiten geschrieben, kaum daß die "hysterischen" Hautkrankheiten etwas eingehender behandelt werden, Hautkrankheiten, die aus der Sucht krank oder doch wenigstens „interessant $\mathrm{zu}^{+}$erscheinen hervorgerufen werden. Sie lassen sich gewöhnlich auf sehr einfache Weise erklären, z. B. die Dermatitis bullosa durch Beträufeln der Haut mit flüssigem Siegellack. Monographien über dieses Thema habe ich leider in der mir zur Verfügung stehenden Literatur nicht gefunden und auch in dem neuesten Buch „Dreuws H. u. G.-Kr. im Kriege und Frieden“" (Berlin, Fischers Med. Buchhandlung) findet die künstliche Hervorrufung von Hautkrankheiten keine Beachtung.

In meiner nun mehr wie einjährigen Praxis als Chefarzt eines k. u. k. Not-Reservespitals mit über 300 BettenBelag und einem regen Wechsel von Verwundeten und Kranken, von welch letzteren ein Großteil von Truppen des Etappenraumes und Hinterlandes stammt, habe ich relativ reichliche Gelegenheit gehabt, Hautkrankheiten zu beobachten, die teils überhaupt künstlich hervorgerufen, teils aber in ihrer Heilungstendenz künstlich gehemmt wurden. Diese Hautkrankheiten waren alle auf den Typus Entzündung zurückzuführen. Die häufigsten Formen waren Dermatitis bullosa, oberflächliche Ulcera cutanea, Ekzeme and Verbrennungen. Der Verdacht, daß es sich um künstlich hervorgerufene oder künstlich erhaltene Hautkrankheiten in den vorliegenden Fällen handelte, war für die klinische Beobachtung hauptsächlich durch zwei Momente gegeben. Das eine Moment, was fast allen gemeinsam war, bestand darin, daß immer Nachschübe auftraten, sobald durch Abheilung des Prozesses die Felddiensttauglichkeit fast erreicht war; und das zweite Moment war der Umstand, daß die Form der Hauterkrankung eine unnatürliche war. Bei 
einem Patienten trat die Reizung der Haut am Rücken strichförmig auf, wie eben die mit dem Reizmittel bestrichenen Finger der Hand heruntergefahren waren. Bei einem andern hatte die Dermatitis haarscharfe, gerade Grenzlinien. Bei einem dritten zeigte eine frischaufgeschossene pralle Hautblase, die für Hautblasen ungewohnte Form eines Rechtecké. Bei einem andern wieder, der mit der Diagnose Ulcera cruris ins Spital geschickt wurde, zeigten die fest und zäh anhaftenden, dicken, schwärzlich verfärbten Schorfe eine ideal kreisrunde Form, zwei von der Größe eines Einkronenstückes, einer von der Größe eines Zweikronenstückes. In der allernächsten Umgebung dieser Schorfe war die Haut entzündlich gerötet. Der Heilungsprozeß verlief analog dem Heilungsprozeß bei Verbrennungen dritten Grades, so daß wohl nach Aussehen der Krankheit und nach dem an ihr beobachteten Verlauf die theoretische Annahme berechtigt erscheint, der Patient habe sich durch Auflegen sehr heißgemachter Geldstücke auf die Hant seines Únterschenkels künstlich seine „Hautkrankbeit" hervorgerufen. Der Patient war einsichtig genug, auf entsprechende Vorstellung die Heilung nicht künstlich zu verzögern. Bei ihrem Eintritt allerdings versuchte er durch Vorschützung einer innerlichen Krankheit seinen Abgang zum Kader hinauszuschieben, was ihm selbstverständlich nicht gelang.

Diese künstlichen Verbrennungen sind natürlich sehr schmerzhaft und finden daher nicht viele Anhänger. Wenigstens war dies der einzige derartige Fall, den ich beobachtete. Die meisten ziehen weniger schmerzhafte Prozeduren vor, um sich eine Hautkrankheit zu verschaffen. Ich glaube, dies wird wohl der Grund sein und nicht eine ideale Vorliebe für das Schöne, daß sich die meisten dieser Drückeberger ihre Mittel zum Krankwerden aus dem Blumenreiche holen. Wir Doktoren der gesamten Heilkunde, die das Studium der Botanik seligen Angedenkens nicht mit jener Gründlichkeit betrieben haben, wie die Kollegen jener Zeiten, wo der Arzt noch Sonntagsspaziergänge benützte, um sich die Heilpflanzen selbst zu pflücken, haben keine Ahnung, welch 
böse Säfte anch die nicht offizinellen duftenden Blumen und Kräuter bergen. Ihre Zahl ist Legion. Jede Jahreszeit läßt sie gedeihen. Für Frühjahr, Sommer und Herbst nimmt uns das nicht Wunder, aber auch im Winter unter Schnee und Eis gedeiht eine Blume, die den poetischen Namen Weihnachtsrose führt, die den Drückebergern hoch willkommen ist, um ihnen, richtig angewandt, als Weihnachtsgabe eine Dermatitis zu bescheren. Eines großen Vertrauens erfreuen sich die verschiedenen Glieder der Familie Hahnenfuh, und viele andre mehr, darunter das so unschuldige Leberblümchen, die einzeln aufzuzählen viel zu viel Raum in Anspruch nehmen würde. Ausgeführt wird die Erzeugung einer Dermatitis auf zweifache Weise. Entweder wird die frische Pflanze verwendet, oder aber der biedere Drückeberger bereitet aus der Pflanze einen spirituösen Extrakt. Zur Bereitung des letzteren darf aber kein zu hochgradiger Alkohol genommen werden, sonst geht die Wirksamkeit des Extraktes in Kürze verloren; so versicherte mir wenigstens ein in diesen Dingen erfahrener Mann. Mit dem Extrakt wird ein Leinwandfleckchen befeuchtet, auf die Haut gelegt, niedergebunden und einige Stunden liegen gelassen. Bei dieser Art der Anwendung wird zweifellos ein Teil der Wirkung dem Alkohol zuzuschreiben sein. Wir wissen ja, daß Alkohol, für längere Zeit auf die Haut gebracht, mit starker Beschränkung der Möglichkeit der Verdunstung, eine Dermatitis zur Folge hat. Die Anwendung der frischen Pflanze z. B. bei der Weihnachtsrose ist die, daß der Stengel zu einem Brei zerdrückt und verrieben wird und dieser Brei nun auf die Haut aufgelegt wird. Die Bedeckung muß aber mit einem impermeablen Stoff erfolgen. Die Leute bedienen sich dazu gewöhnlich des Pergamentpapiers. Nimmt ein Unerfahrener in seiner Unschuld ein Leinwandfleckchen, so ist der Erfolg fraglich, da sich die Flüssigkeit des Breis und damit auch die Wirksamkeit der Substanz in das Leinwandfleckchen aufsangt. Die Weihnachtsrose wirkt relativ milde und benötigt mehrere Stunden, um die gewünschte Dermatitis hervorzurufen. Rascher geht es beim Hahnenfuß. Schon 
nach wenigen Minuten bildet sich an der Stelle, wo er anfgelegt war, ein roter Fleck und eine Blase. Wird die Blase geöffnet und die Sache in Ruhe gelassen, so ist der Prozeb in 8-10 Tagen zur Abheilung gekommen. Wenn man aber als wohlwollender Arzt dem Patienten durch Salbenverband etc. Linderung bringen will, so vergehen 3-4 Wochen, bis sich wieder normale Haut über dem oberflächlichen Substanzverlust gebildet hat. Daß Fett eine Verzögerung in der Heilung bewirkt, wissen viele Drückeberger. Sie verwenden diese Erfahrung. Auf die nur gerötete oder durch Blasenzerstörung epidermislos gemachte Haut bringen sie ein Scheibchen rohen Speckes (rohes Fleisch soll auch gute Dienste tun), binden es fest und lassen es eine Nacht liegen. Und am andern Tage ist ihre Arbeit belohnt durch einen oberflächlichen, eiternden Substanzverlust. In ähnlicher Weise werden natürlich noch viele andre Mittel verwendet, so ranziges Fett, Senfgeist etc. etc. Der Senfgeist ist sehr beliebt, um oberflächliche Geschwüre im Stadium der Abheilung wieder zur Exazerbation zu bringen. Es bilden sich da um die in Epithelisierung begriffene Wunde auf mäßig gerötetem Grunde matsche Blasen, die bei entsprechender, leicht durchführbarer traumatischer Mißhandlung zu einem Wiederaufflackern und räumlichen Fortschreiten der Ulzeration Veranlassung geben.

Es besteht eine Verordnung, daß bei Hauterkrankungen, welche den Verdacht erwecken, künstlich hervorgerufen worden zu sein, dem Patienten über die erkrankten Stellen ein fester Verband angelegt werde, Gips oder Blaubinden, damit er nicht in die Lage kommen könne, seine kranken Hautstellen zu reizen und ihre ruhige Abheilung zu verhindern. Der Drückeberger weiß sich zu helfen. Unter dem noch feuchten Verband z. B. am Unterschenkel, läßt er seine Muskeln spielen; wenn es ihm möglich ist, geht er auf und ab, stellt sich auf die Zehen; wenn er vorsichtshalber zu Bett gebracht, aber nicht genügend beaufsichtigt ist, so bewegt er den Fuß extrem wie nur möglich. Durch diese Manipulationen bezweckt er und erreicht er eine Dehnung des Verbandes, so daß derselbe dann, erstarrt, nicht enge 
den Unterschenkel umschließt. Nun fährt er mit einem feinen Stäbchen zwischen Verband und Haut von oben nach unten durch, befestigt unten mittelst eines Fadens ein Leinwandläppchen, das er mit einer reizenden Substanz getränkt hat, bringt es durch Zurückziehen des Stäbchens an die gewünschte Stelle, läßt das reizende Mittel nach seiner Erfahrung entsprechend lange wirken und entfernt dann dasselbe durch völliges Herausziehen. Bei Öffnung des Verbandes hat man dann die unangenehme Überraschung, daß die Ulzeration nicht nur nicht besser, sondern im Gegenteil an Ausdehnung gewonnen hat. Geht der Verband auch über den ganzen $F u ß$, so daß er mit seinem Unterschenkel wie in einem gutsitzenden Stiefel steckt, so bemüht sich der Schwindler, so lange der Verband feucht ist, eine möglichst unnatürliche Fußhaltung einzunehmen, bis der Verband fest geworden ist. Wenn er nun den Fuß nicht mehr in der unnatürlichen, verdrehten Stellung hält, sondern in die normale Lage zurückgehen lassen will, so kann das der Fuß nicht, jetzt reizt der nun fest gewordene Verband den Fuß an der den normalen Verhältnissen des Fußes nicht passenden Stelle.

Ich glaube, es ist nicht ünwichtig darauf hinzuweisen, daß auch auf dem Wege der Hautkrankheiten Leute sich ihren staatsbürgerlichen, militärischen Pflichten entziehen wollen. Wenn die Zahl derselben auch lange nicht jene erreicht, welche andre Krankheiten, wie z. B. den mit Recht so beliebten Rheumatismus vorschützen, so ist es schließlich bei einer Armee, die nach Millionen zählt, doch eine erkleckliche Anzahl. Wir Ärzte sind berufen und haben die heilige Pflicht, nach unsrem besten Wissen und Gewissen ohne falsche Gefühlsduselei derartige Schwindler so rasch wie möglich ihrer Pflicht zurückzuführen, wenn sie aber widerhaarig sind, sie unnachsichtlich dem Arme der Gerechtigkeit zu überliefern, denn es gibt nichts Gemeineres, nichts Niedrigeres als schamlose Drückebergerei, während Tausende ohne Murren fürs Vaterland und für ihr Volk das Leben lassen. 


\section{$B$. Erysipel.}

Obwohl der Rotlauf seiner ganzen Ätiologie und Histologie nach sich wie ein Paradigma der Hautkrankheiten darstellt, ist en doch in den letzten Jahren dem Besitzstande der Dermatologie mehr weniger verloren gegangen. Erst der Krieg gibt uns wieder mehr Gelegenheit, Fäle von Erysipel zu sehen. Als Therapie hat sich mir weitaus am besten eine kombinierte Behandlung von Jodtinktur äußerlich und Chinin innerlich bewährt. Holländer und Oppenheim haben diese Art der Behandlung für Lupus erythematosus mit großem Erfolg eingeführt. Bei ihrer Anwendung für das Erysipel ging ich von der Voraussetzung aus, daß gerade bei dieser Krankheit, wo, wie wir wissen, der Krankheitserreger der Streptokokkus Fehleisen sich in den Lymphgefäßen und Saftkanälchen der Haut ausbreitet, eine lokale Behandlung der erkrankten Hautstellen mit Tiefenwirkung von großem Nutzen sein müsse, weil sie ja gewissermaßen eine kausale Therapie darstellt. Die usuellen lokalen Behandlungsmethoden beschränken sich entweder auf rein symptomatische Erfolge, oder aber sie können vernöge ihrer geringen Tiefenwirkung nicht bis zu den Nestern des Krankheitserregers vordringen. Der praktische Erfolg hat den theoretischen Erwägungen Recht gegeben. Nach zweifacher Richtung kann ich einen Vorteil gegenüber den andern Behandlungsmethoden feststellen. Erstens gelingt es in den meisten Fällen das Fortschreiten der Krankheit einzuschränken, zweitens ist bei allen Krankheitsfällen die Krankheitsdauer beträchtlich abgekürzt. Ich setze der Jodtinktur $1-2 \%$ acid. carbol. liquef. bei und lasse mit dieser Mischung die erkrankten Stellen nach vorheriger Reinigung mit Watte und Benzin oder Watte mit Äther ein- bis zweimal täglich tüchtig einpinseln, dabei Bedacht nehmend, daß die Bepinselung gut $2 \mathrm{~cm}$ in die scheinbar gesunde Haut hinüberreicht. Fulls die Haut nach einigen Bepinselungen starke Tendenz zum Rissigwerden zeigt, lasse ich der Jod-Karboltinktur einige Tropfen Glyzerin beisetzen. Bei Erysip. bullos. müssen die Blasen möglichst rasch weggebracht werden, durch Bepudern oder durch 
Ein Beitrag zum Kapitel: Dermatologische Kriegserfahrungen. 291

Einstechen je nach ihrer Größe. Der Blasengrund wird dann genau so behandelt wie die andere erkrankte Haut. Gewöhnlich genũgt eine Gabe von $0.1-0.2 \mathrm{~g}$ Chinin mur. dreimal täglich gegeben. Bei sehr hohem Fieber oder bei einer ausgesprochenen Tendenz zum Weiterwandern des Erysipels steige ich bis dreimal täglich $0.5 \mathrm{~g}$ und habe damit immer das Auslangen gefunden. Die leichten unangenehmen Nebenerscheinungen des Chinins werden vom Patienten und vom Arzt gerne mit in den Kauf genommen.

\section{Erfrierungen.}

Die Karpathenkämpfe haben auch in unser Spital zahlreiche Fälle von Erfrierungen geliefert. Der Ausdehnung nach und dem Grade nach waren dieselben sehr verschieden. In der Behandlung befolgten wir die von autorativer Seite gegebene Richtschnur „bis zum Extrem konservativ". Der Erfolg war ein großer trotz schwerster Zerstörungen. Für Erfrierungen leichten Grades bedienten wir uns einer Salbe, die den Vorteil hat, in ihrer Herstellung sich billig za stellen und doch sehr gute Ergebnisse zu liefern. Die Formel für die Salbe lautet:

Extr. hippocast. 10.0,

Ung. plumb. acet. $90^{\circ} 0$.

\section{$D$. Gonorrhoe und Lues.}

In unserem Notreservespitale waren bis nun über 2000 Soldaten als Patienten. Jeder unserer Spitalspfleglinge wird während seines Spitalsaufenthaltes je nach der Länge desselben ein- oder mehrmals quoad Infektion mit einer Geschlechtskrankheit untersucht, besonders genau aber anläßlich der Entlassung aus der Spitalsbehandlung. Trotzdem die Patienten reichlich Ausgang haben, habe ich bisher keinen einzigen Fall von frischer Infektion mit Gonorrhoe und nur einen Fall von Ulcus durum konstatieren können; ob diese günstige Statistik auf einen Zufall zurückzuführen ist oder auf Belehrung, welche allerdings nicht schulmeisterlich, sondern mehr in scherzhafter, oft auch derber Weise gegeben wurde, entzieht sich meiner Entscheidung. Auch die Zahl der Fälle subakuter und chronischer 
Gonorrhoe sowie von Lues, deren Infektion vor der Spitalsaufnahme lag, war im Verhältnis zur Spitalsfrequenz keine abnorm hohe. Anders in der Privatpraxis. Da läßt sich zweifellos ein Anschwellen der Infektionen mit Geschlechtskrankheiten feststellen; hiebei ist nach meiner Erfahrung die Gonorrhoe der kleinere Bruder der Lues.

Die vorurteilslose Beobachtung lehrt, daß für die $\mathrm{Zu}-$ nalume der Luesfälle unzweifelhaft das Abweichen von der bewährten Quecksilberbehandlung mitverantwortlich zu machen ist. Schon zu Friedenszeiten ließ sich beobachten, daß Leute, welche das Unglück hatten Lues zu akquirieren und das noch größere in die Behandlung eines unbedingten Salvarsananhängers zu treten, nicht so selten zur ausgiebigen Quelle von neuen Luesinfektionen wurden. Natürlich ohne Absicht und gegen ihren Willen. Nirim dein Bett und geh! Laß dir eine oder zwei Salvarsaninjektionen machen und du bist gesund! Niemand glaubt dies lieber wie der Kranke, insbesonders der Lueskranke, der ja doch so vom Hörensagen aus dem Kreise seiner Freunde und Bekannten ahnt, wie langwierig diese Kraukheit verläuft, welch' gewissenhafter Befolgung der ärztlichen Ratschläge es bedarf, um sie endlich los $z u$ werden.

Diese Versicherung von ärztlicher Seite, so rasch zu genesen, hebt selbstverständlich das Wohl- und Kraftgefühl der Patienten, nicht in letzter Linie auch in der sexuellen Sphäre. Was ist die natürliche Folge davon? Während der Patient früher unter dem drückenden Bewußtsein, für lange sexuell krank zu sein, den Ermahnungen des Arztes, sich jeden sexuellen Verkehres zu enthalten, willig, ja bußfertig unterwarf, verleitet ihn jetzt die Vorstellung: "wenn mir wieder was passiert, unterziehe ich mich halt noch einer Salvarsankur", sich in sexueller Beziehung keiner oder nur sehr vorübergehender Beschränkung zu unterziehen.

Ein Beispiel der Praxis bestätige die Richtigkeit dieser Ausführung: Ein Herr mit frisch akquirierter Lues tritt in meine Behandlung. Es wird eine energische $\mathrm{Hg}$-Kur eingeleitet. Der Patient $m u ß$ geschäftlich verreisen. Auf die Reise wird ihm die Ermahnung mitgegeben, die Kur nicht 
zu unterbrechen. Nach einigen Monaten sucht er wieder meine Sprechstunde auf. Am Penis blühen Papeln. Und nun erzählt er: In einer großen Stadt sei er meinem Auftrage entsprechend zu einem Arzte gegangen, um sich eine $\mathrm{Hg}$ Salizylinjektion machen zu lassen. Der Arzt habe ihn aber überredet, doch lieber zwei Salvarsaninjektionen zu nehmen; da sei er dann vollkommen gesund. Der Einwand des Patienten, daß ihm sein ursprünglicher Arzt Hg-Injektionen als verläßlichstes Mittel empfohlen habe, wird mit der ziemlich verächtlich gegebenen Bemerkung; "das sei halt ein älterer Arzt", niedergekämpft.

Nun steht der bedauernswerte Patient da! Im guten und vor allem angenehmen Glauben an die Versicherung: des Kollegen hat er sich nach den Salvarsaninjektionen nicht weiter um seine Lues gekümmert, flott darauf losgelebt und kaum heimgekehrt, mit seinen Papeln die Frau infiziert. $\mathrm{Da}$ er auch während der Reise kein sexueller Kostverächter war und, wie er selbst zugestand, „diese Geschichte", d. s. die Papeln, schon länger bestanden, sicher reichlich für die Verbreitung der Lues gesorgt.

Es müssen doch alle Ärzte, wenn sie willig sind zu sehen, über ähnliche Erfahrungen verfügen!

Trotzdem wurde in den letzten Monaten von verschiedenen Seiten der Vorschlag gemacht, für die Behandlung. der, an Lues erkrankten Soldaten in der Front und hinter der Front als Panazee offiziell eine ambulatorische Salvarsanbehandlung einzuführen, so nach Art der Schnellsohlereien, wobei sich, nach dem oben gesagten, das tertium comperationis sicher auch auf die Dauerwirkung der "Heilung" erstreckt.

Dazu kommen noch die so unerwünschten und so gefährlichen Nebenwirkungen des Salvarsans als solchen, welche trotz aller Spitzfindigkeiten kein vernünftiger $\mathrm{Be}$ obachter ableugnet, vermehrt um die schädigenden Nebenwirkungen, welche durch die oft mangelhafte Technik der Injektion bedingt sind. Die Fälle sind nicht so selten, in welchen auch durch letztere nach Salvarsaninjektionen die angestrebte Kriegsdiensttauglichkeit sehr verzögert wurde. 